\title{
VCPS Vehicle Connect Performance Study
}

\author{
Meifeng Xiao ${ }^{\text {a) }}$ and Lichen Zhang ${ }^{\text {b) }}$ \\ School of Computer, Guangdong University of Technology, Guangdong 510006, China \\ a) Corresponding author: 2441015805@qq.com \\ b) Zhanglichen1962@163.com
}

\begin{abstract}
The Cyber Physical System, as an emerging product of embedded systems, sensor networks, and wireless networks, has received extensive attention from domestic and foreign scholars. Lots of Cyber Physical System have emerged, such as medical CPS and energy CPS. And so on, VCPS is one of the applications. In VCPS, due to the continuous increase in the number of vehicles, road safety accidents, traffic congestion, fuel consumption and other phenomena have become more serious. Vehicle communication is a very important part of information transmission in VCPS, but due to the characteristics of high-speed driving of vehicles, the time for establishing connection of vehicles is very short and the network topology changes dynamically. Therefore, it is also a great challenge how to ensure the vehicle communication quality between vehicles traveling at a high speed. This dissertation focuses on the connection performance of vehicles, and investigates the connectivity analysis of vehicles, the dynamic spectrum access of cognitive radio and the method of VANET adaptive connection parameters.
\end{abstract}

Key words: CPS; Cyber Physical System; VCPS; VANET; Vehicle communication.

\section{INTRODUCTION}

Traffic accidents and traffic congestion continue to deteriorate worldwide. With the increasing number of vehicles each year, road traffic pressure is huge, resulting in a series of adverse effects such as traffic accidents, traffic jams, fuel wastage, and transportation safety. The VPS (Vehicular Cyber Physical System) aims to solve this problem. It is a subset of CPS. It is the application of CPS in road traffic, mainly combining calculation, communication and control. Vehicle-to-vehicle, vehicle-to-roadside communications will provide vehicle drivers or self-driving vehicles with vehicle information in a timely manner to reduce traffic congestion, accidents, and fuel consumption on the road. In VCPS, the connection is considered to be the pillar of intelligent VCPS communication. It can provide timely information to the driver or provide feedback to vehicles on the road to improve road safety and overall traffic efficiency.

\section{VCPS COMMUNICATION METHOD}

In VCPS, the communication modes are Vehicle to Vehicle (V2V), Vehicle to Roadside Unit (V2R), Roadside Unit to Vehicle (R2V), Roadside Unit to Roadside Unit (R2R) [1], as shown in Figure 1.

In V2V communication, vehicles use single-hop or multi-hop information exchange instead of fixed roadside units. This vehicle communication does not require any roadside infrastructure. Therefore, it applies to places and emergencies where communication infrastructure cannot communicate.

In V2R communications, vehicles rely on roadside infrastructure (such as base stations or access points) for information exchange, and roadside infrastructure for $\mathrm{V} 2 \mathrm{R}$ and $\mathrm{R} 2 \mathrm{~V}$ communications and potential R2R communications. In such communications, roadside infrastructure plays an important role in the connectivity and security of vehicle communications [2-4]. Roadside infrastructure can verify the identity of the vehicle to provide VCPS security. In addition, roadside infrastructure helps connect disconnected vehicles when vehicle density is low. 
However, V2R and R2V communications do not apply to time-sensitive emergency communications because of the large delays they cause.

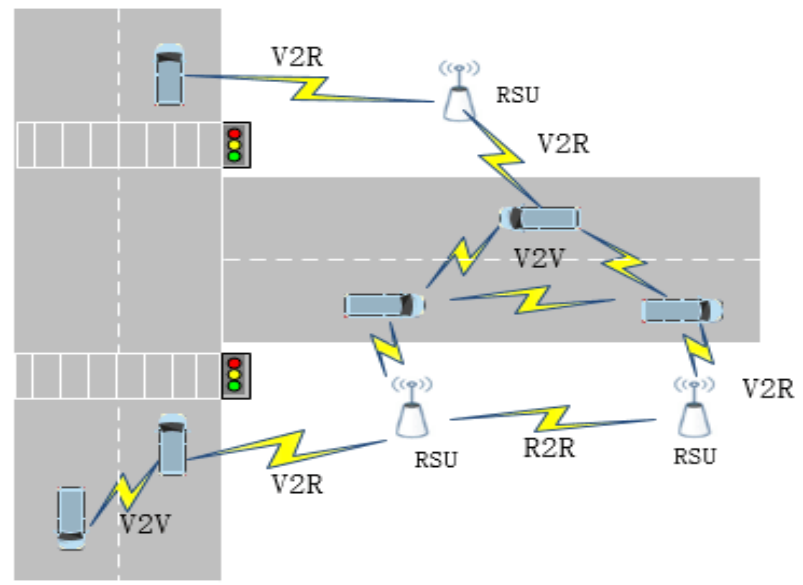

FIGURE 1. Vehicle network communication

\section{VEHICLE CONNECTIVITY ANALYSIS}

The connectivity between vehicles is very important, especially for disseminating upcoming traffic information (such as vehicle accident warning information, etc.) to other follow-up vehicles in order to promptly inform the driver to take appropriate preventive measures. Vehicle networking is the Intelligent Transportation System (ITS) to improve passenger comfort, traffic efficiency, and passenger safety by sending upcoming traffic information in a timely manner. One of the main goals of wireless communications in VANET is the automation of message propagation using vehicle-to-vehicle $(\mathrm{V} 2 \mathrm{~V})$ and/or vehicle-to-roadside (V2R) and possibly curbside to roadside (R2R) communications. In V2R communication, the vehicle sends information to the roadside unit and the roadside unit propagates the information to the target vehicle. This process can cover a large area, but because the roadside unit acts as a relay between the sender and the receiver, it introduces delays in message propagation. This type of communication is not suitable for time emergency or emergency messages. In V2V communication, each vehicle serves as a source, destination, and/or router to propagate traffic related information. Therefore, using V2V communication can help reduce the delay introduced by roadside units. In addition, most safety-related applications (such as collision warnings, merge roads, emergency braking, etc.) require the use of short-range V2V communications for fast message exchange.

There are two problems in the connection of vehicles. The first is due to the highly dynamic VANET topology and the short transmission range of Dedicated Short-Range Communication (DSRC) stipulated by the Federal Communications Commission (FCC). The communication link between vehicles is short-lived, especially in the case of sparse vehicle density, it is difficult to maintain rapid vehicle communication. When a fixed transmission range is used when the VANET is dynamically changed, the network may be easily affected by a "broadcast storm," and vehicles in the VANET may be frequently disconnected when the vehicle density is low. At the same time, when using high frequency and wireless transmission range/power, broadcast storm problems may occur due to replay of messages from multiple vehicles within a given transmission range. The second is the problem of transmission delay of time-sensitive emergency messages [5]. Equal treatment of messages will result in sensitive emergent information being at risk of high delay. Therefore, vehicles should be able to adapt their communication parameters, including transmit power/range, etc. according to their respective local environment. The message difference becomes an important method for quickly transmitting time-sensitive emergency messages in the network. The vehicle network topology constantly changes according to the speed of the vehicle and the behavior of the driver. Maintaining the connectivity of this dynamic network topology is one of the challenges of VCPS. The following provides some research progress and research contents on the improvement of the connection performance of the vehicle, mainly from the analysis of the connectivity of the vehicle and the analysis of the VANET adaptive connection: 
One of the main goals of wireless communication in VANET is the use of vehicle-to-vehicle (V2V), vehicle-toroadside unit (V2R), and possibly roadside unit to roadside unit (R2R) communications for automated message propagation. In V2R communication, the vehicle sends information to the roadside unit and the roadside unit sends the information to the target vehicle. This process can cover a large area, but because the roadside unit acts as a relay between the sender and the receiver, it introduces delays in message propagation. This type of communication is not suitable for time-critical communications or emergency messages. In V2V communication, vehicles act as senders, receivers, or routers to propagate traffic-related information, reducing the delays caused by intermediate transmissions in the V2R communication process. Therefore, using V2V communication can help reduce the delay introduced by roadside units. In addition, most safety-related applications (such as collision warnings, merge roads, emergency braking, etc.) require the use of short-range V2V communications for fast message exchange. However, due to the high speed of the vehicle, the connectivity of the vehicle is difficult to be continuously stabilized, and the performance of improving the vehicle connectivity is very important in the vehicle communication. Literature [6] studied the influence of the correlation time, vehicle's relative speed, transmission distance and data size on communication performance in short-distance $\mathrm{V} 2 \mathrm{~V}$ communication. [7, 8] describes connectivity in onedimensional VANET. The analysis of connectivity-aware routing in VANET is described in [9, 10]. In order to improve connectivity, an additional vehicle was introduced in [8] as a mobile base station (BS) and a roadside base station was used in [11]. Without considering the impact of the dynamic adaptation of the vehicle's stochastic speed and transmission range, the literature [12] proposes connectivity analysis for unidirectional and bidirectional road scenarios combined with channel fading, propagation path loss, transmission power, and transmission data rate. The deficiencies in the literature [9] were optimized.

\section{ADAPTIVE VANET PARAMETERS}

One strategy for increasing the duration of communication links in VANET is to increase the transmission range in the case of sparse traffic density but increasing the transmission range may result in high levels of destructive interference and high network overhead under high-density communication conditions. Along with this, the transmission power that dynamically adapts to changing vehicle density is a key requirement in VANET. In addition, in order to disseminate emergency information in a timely manner, VANET must support some form of message differentiation. In the IEEE 802.11e contention-based channel access mechanism EDCA [13], to achieve this strategy, different priorities can be assigned to various vehicle-related messages according to their urgency or delay requirements. For example, news related to road accidents should be accurately transmitted to the target area on time to avoid congestion and potential secondary accidents.

Suthaputchakun and Ganz [14] studied security messages using IEEE 802.11e as an inter-vehicle communication (IVC) in priority-based vehicle-based ad hoc networks (VANET). Message priority based on message urgency allocation correlates with different quality of service in terms of average delay and normalized throughput. Use IEEE $802.11 \mathrm{e}$ to provide priority-based service differentiation. In order to improve the reliability of communications, they also use a repetitive transmission mechanism to provide a reliable differential ratio for each priority message and incorporate the 802.11e EDCA mechanism. However, it does not consider the adaptation of QoS parameters, nor does it consider the adaptation of transmission power. The literature [15] discusses the adaptive transmission power in VANET based on vehicle density, and Balon and Guo [16] discuss the CW size problem of dynamic adaptive broadcast in VANET. However, the author of [16] only considered the channel access time based on the urgency of the message and its delay requirement, without considering the adaptability of the transmission power.

Literature [17] proposed a scheme for dynamically adapting the transmission power and contention window (CW) size to improve the performance of information dissemination in vehicle self-organizing networks (VANET). The proposed scheme includes an 802.11e enhanced distributed channel access (EDCA) mechanism and uses a joint approach to adapt the physical layer (PHY) transmission power and the media access control layer (MAC) quality of service (QoS). In the solution, the transmission power is adapted based on the estimated local vehicle density to dynamically change the transmission range, and the $\mathrm{CW}$ size is adjusted according to the data collision rate. To facilitate the timely dissemination of information, VANET's visits are prioritized according to their urgency and disseminated using the EDCA mechanism. Different from the method proposed by Artimy [15]. Simulation results show that the solution has better performance. 


\section{CONCLUSION}

Firstly, this paper puts forward the importance of vehicle communication quality and analyzes some research methods and research results to improve the vehicle communication quality. At the same time, the technology of self-adaptive connection parameters of vehicles is studied. The methods of adaptive transmission power, transmission range, local vehicle density, data collision rate, contention window size and other parameters are analyzed to improve the throughput of communication and low delay of messages.

\section{ACKNOWLEDGMENTS}

This work is supported by the national natural science foundation of China under grant (No.61572142), natural science foundation of Guangdong province under grant (No.2015A030313490).

\section{REFERENCES}

1. D.B. Rawat, C. Bajracharya, "Vehicular Cyber Physical Systems", Springer (2017).

2. Lee J, Bagheri B, Kao H A. "A Cyber-Physical Systems architecture for Industry 4.0-based manufacturing system”. Manufacturing Letters, 2015, 3:18-23.

3. Rajkumar R, Lee I, Sha L, et al. "Cyber-physical systems: The next computing revolution", Design Automation Conference. IEEE, 2010:731-736.

4. Li Zhuopeng, Zhang Tianchi, Zhang Jing. "Survey on the Research of Cyber-Physical Systems (CPS)". Computer science, 2011, 38(9):25-31.

5. Wu Libing, Fan Jing, Wang Jing, et al. "Emergency Message Broadcast Method Based on Huffman-Like Coding". Computer research and development, 2017, 54(11):2475-2486.

6. Rawat D B, Bista B B, Yan G, et al. "Vehicle-to-Vehicle Connectivity and Communication Framework for Vehicular Ad-Hoc Networks", Eighth International Conference on Complex, Intelligent and Software Intensive Systems. IEEE, 2014:44-49.

7. Yousefi S, Altman E, El-Azouzi R, et al. "Improving connectivity in vehicular ad hoc networks: An analytical study". Computer Communications, 2008, 31(9):1653-1659.

8. Chandrasekharamenon N P, Ancharev B. "Connectivity analysis of one-dimensional vehicular ad hoc networks in fading channels". Eurasip Journal on Wireless Communications \& Networking, 2012, 2012(1):1.

9. Yan G, Olariu S. "A Probabilistic Analysis of Link Duration in Vehicular Ad Hoc Networks". IEEE Transactions on Intelligent Transportation Systems, 2011, 12(4):1227-1236.

10. Naumov V, Gross T R. "Connectivity-Aware Routing (CAR) in Vehicular Ad-hoc Networks", IEEE INFOCOM 2007 -, IEEE International Conference on Computer Communications. IEEE Computer Society, 2007:1919-1927.

11. Sou S I, Tonguz O K. "Enhancing VANET Connectivity through Roadside Units on Highways", IEEE Transactions on Vehicular Technology, 2011, 60(8):3586-3602.

12. Panichpapiboon S, Pattara-Atikom W. "Connectivity Requirements for Self-Organizing Traffic Information Systems”. IEEE Transactions on Vehicular Technology, 2008, 57(6):3333-3340.

13. Part 11: Wireless LAN Medium Access Control (MAC) and Physical Layer (PHY) Specifications Amendment 8: Medium Access Control (MAC) Quality of Service Enhancements, IEEE Std. 802.11e, 2005.

14. Suthaputchakun C, Ganz A. "Priority Based Inter-Vehicle Communication in Vehicular Ad-Hoc Networks using IEEE 802.11e", IEEE, Vehicular Technology Conference - Vtc2007-Spring. IEEE, 2007:2595-2599.

15. Artimy M. "Local Density Estimation and Dynamic Transmission-Range Assignment in Vehicular Ad Hoc Networks". IEEE Transactions on Intelligent Transportation Systems, 2007, 8(3):400-412.

16. Balon N, Guo J. "Increasing broadcast reliability in vehicular ad hoc networks", International Workshop on Vehicular Ad Hoc Networks, Vanet 2006, Los Angeles, Ca, Usa, September. DBLP, 2006:104-105.

17. Rawat D B, Yan G, Popescu D C, et al. "Dynamic Adaptation of Joint Transmission Power and Contention Window in VANET", Vehicular Technology Conference Fall. IEEE, 2010:1-5. 